\title{
Mean-square global exponential stability in Lagrange sense for delayed recurrent neural networks with Markovian switching
}

\author{
Qiuxin Chen ${ }^{1}$, Lei Liu ${ }^{1, *}$, Ailong $\mathrm{Wu}^{2}$ \\ ${ }^{1}$ College of Science, Hohai University, Nanjing 210098, China \\ ${ }^{2}$ College of Mathematics and Statistics, Hubei normal university, Huangshi 435002, China
}

\begin{abstract}
The paper discusses the mean-square global exponential stability in Lagrange sense for delayed recurrent neural networks (DRNNs) with Markovian switching. Two different types of activation functions are considered, which include both bounded and unbounded activation functions. By using the vector Lyapunov function and stochastic analysis technique, we establish two $\mathcal{L}$-operator differential inequalities. By employing the two $\mathcal{L}$-operator differential inequalities and vector Lyapunov functions methods, we provide easily verifiable criteria for Lagrange stability in mean-square sense of DRNNs with Markovian switching. Finally, two numerical examples are given to illustrate the efficiency of the derived results.
\end{abstract}

Keywords: Delayed recurrent neural networks, Mean-square exponential stability, Lagrange stability, Global exponential attractive, Markovian chain, M-matrix

\section{Introduction}

The recurrent neural networks (RNNs) have been applied in many fields to solve optimization, control and signal processing problems, since the pioneering work of Hopfield [1]. A large number of researches now exist on the study of stability theory for RNNs [2]-[15]. One of the most desirable properties of RNNs is the Lyapunov global stability. From a dynamical system point of view, global stable networks in Lyapunov sense are monostable systems, which have a unique equilibrium attracting all trajectories asymptotically. On the other hand, monostable neural networks have been found computationally restrictive and multistable dynamics are essential to deal with important neural computations desired. For example, in a winner-take-all network $[9,16]$, where, depending on the external input (or the initial value), only the neuron with the strongest input (or highest initial value) should remain active. This is possible only if there are multiple equilibria with some being unstable. As we know, Lagrange stability is one of the most important properties in multi-stability analysis, and has been extensively studied by many researchers $[3,5,8]$, [17]-[20]. For example, Liao et al. generalized the conventional notion of stability in Lyapunov sense and studied the global exponential stability (GES) in Lagrange sense for DRNNs in [3]. Then, Tu et al. extended the results of Liao et al. in [3], by using linear matrix inequality(LMI) [8]. It is worth mentioning that Lagrange stability refers to the stability of the total system which doesn't require the information of equilibrium points. Hence, the global stability in a Lyapunov sense can be viewed as a special case of stability in a Lagrange sense by regarding an equilibrium point as an attractive set [21].

In modeling realistic problems, time-varying delay comes closer to reflecting the realistic model [22, 23]. While effective, the obtained results in $[3,24,25]$ are only applicable to the constant delay systems. Although some authors have focused on the Lagrange stability analysis for DRNNs [17]-[19], one important restrictive condition that the derivative value of time varying delay is less than one must be imposed. To the best of our knowledge, without the

\footnotetext{
${ }^{*}$ Corresponding Author.

E-mail addresses: liulei_hust@hhu.edu.cn(L. Liu)
} 
restrictive condition that the derivative value of time-varying delay is less than one, the Lagrange stability analysis for neutral networks with time-varying delay has scarcely been investigated. Thus, tackling this issue constitutes the first motivation of this paper.

Meanwhile, hybrid systems driven by continuous-time Markovian chains are widely employed to model real-life systems that may experience abrupt changes in system structure and parameters, such as battle management command, control, and communication systems, failure prone manufacturing, system-on-chip hardware design verification, ship steering, channel identification, population dynamics [26]-[32]. On the other hand, it is well known that neural networks can be represented by a switching model which can be regarded as a set of parametric configurations switching from one to another according to a given rule. Recently, a large number of scholars have studied the stability of Markovian switching networks, such as the robust stability and synchronization of Markovian switching networks [33]-[35]. And the exponential stability of Markovian switching neural networks was analyzed in [6, 36].

However, as far as we know, few authors have discussed the stability in Lagrange sense of DRNNs with Markovian switching. The existing techniques proposed in [3,8] cannot be easily used for the Lagrange stability analysis of DRNNs with Markovian switching, due to the existence of Makovian switching mechanism in delayed systems, which may make the problem complex and difficult. Solving this problem is the second motivation of this paper.

Motivated by the above analysis, in this paper, we will study the global exponential stability in Lagrange sense for DRNNs with Markovian switching. To this end, we generalize the conventional notion of stability in Lagrange sense and establish the mean-square global exponential stability in Lagrange sense for DRNNs with Markovian switching. We provide easily verifiable criteria for the boundedness of the networks and the existence of globally mean-square attractive sets by constructing appropriate vector Lyapunov functions, which has several obvious advantages over scalar Lyapunov function. Meanwhile, we consider bounded continuous functions and Lurie-type of activation functions, which include both bounded and unbounded activation functions.

The main contributions of this paper lie in three aspects as follows. Firstly, the notion of stability in Lagrange sense for DRNNs can be generalized. Secondly, based on the two $\mathcal{L}$-operator differential inequalities, the global exponential stability in Lagrange sense for delayed recurrent neural networks with Markovian switching can be investigated. Finally, several sufficient criteria for mean-square global exponential stability in Lagrange sense of DRNNs with Markovian switching can be obtained.

\section{Notation}

Throughout this paper, unless otherwise specified, let $\left(\Omega, \mathscr{F},\left\{\mathscr{F}_{t}\right\}_{t \geq 0}, \mathbb{P}\right)$ be a complete probability space with a filtration $\left\{\mathscr{F}_{t}\right\}_{t \geq 0}$ satisfying the usual conditions (i.e., it is increasing and right continuous while $\mathscr{F}_{0}$ contains all $\mathbb{P}$-null sets). Let $\tau>0$, and denote $C\left([-\tau, 0] ; \mathbb{R}^{n}\right)$ (or simply denoted by $C$ ) the family of continuous functions $\varphi$ from $[-\tau, 0]$ to $\mathbb{R}^{n}$ with the norm $\|\varphi\|=\sup _{-\tau \leq \theta \leq 0}|\varphi(\theta)|<\infty$, where $|\cdot|$ is the Euclidean norm in $\mathbb{R}^{n}$. For a given constant $\mathcal{H}>0$, $C(\mathcal{H})$ is defined as the subset $\{\varphi \in C:\|\varphi\|<\mathcal{H}\}$. Let $\Pi$ be the set of all nonnegative functionals $\mathcal{K}: C \rightarrow[0, \infty)$, mapping bounded sets in $C$ into bounded sets in $[0, \infty)$. Let $G$ is a vector or matrix. By $G \geq 0$ we mean each element of $G$ is non-negative. By $G \gg 0$ we mean all elements of $G$ are positive. If $G_{1}$ and $G_{2}$ are vectors or matrices with same dimensions we write $G_{1} \geq G_{2}, G_{1} \gg G_{2}$ if and only if $G_{1}-G_{2} \geq 0, G_{1}-G_{2} \gg 0$, respectively. And if $G=\left(g_{i j}\right)_{n \times n} \in R^{n \times n}$, we denote $|G|=\left(\left|g_{i j}\right|\right)_{n \times n}, \bar{G}=\left(\bar{g}_{i j}\right)_{n \times n}$ with $\bar{g}_{i j}=g_{i j}(i \neq j), \bar{g}_{i i}=g_{i i}^{+}=\max \left\{g_{i i}, 0\right\}, i=1, \cdots, n$, denoted by $\lambda_{\max }(G)$ and $\lambda_{\min }(G)$ as its largest and smallest eigenvalue, respectively. Moreover, we also adopt here the traditional notation by letting

$$
Z^{n \times n}=\left\{A=\left(a_{i j}\right)_{n \times n}: a_{i j} \leq 0, i \neq j\right\} .
$$

Let $r(t), t \geq 0$, be a right-continuous Markovian chain on the probability space taking values in a finite state space $\mathcal{M}=\{1,2, \ldots, \mathrm{N}\}$ with generator $\Gamma=\left(\gamma_{i j}\right)_{\mathrm{N} \times \mathrm{N}}$ given by

$$
P\{r(t+\Delta)=j \mid r(t)=i\}= \begin{cases}\gamma_{i j} \Delta+o(\Delta) & i \neq j \\ 1+\gamma_{i j} \Delta+o(\Delta) & i=j,\end{cases}
$$

where $\Delta>0$. Here $\gamma_{i j}>0$ is transition rate from $i$ to $j$ if $i \neq j$ while $\gamma_{i i}=-\sum_{i \neq j} \gamma_{i j}<0$.

Let us consider a DRNN with Markovian switching

$$
\dot{x}(t)=-D(r(t)) x(t)+A(r(t)) f(x(t))+B(r(t)) f(x(t-\tau(t)))+U(r(t)),
$$


on $t \geq 0$ with initial data $\varphi \in C$, where $x(t)=\left(x_{1}(t), \cdots, x_{n}(t)\right)^{T}$ is the state of the neuron, $D(l)=\operatorname{diag}\left(d_{1}(l), \cdots, d_{n}(l)\right)$ is a self-feedback connection weight matrix, $A(l)=\left(a_{i j}(l)\right)_{n \times n}$ and $B(l)=\left(b_{i j}(l)\right)_{n \times n}$ are, respectively, connection weight matrices associated without delay and with delay, respectively. For $i=1,2, \cdots, n, \tau_{i}(t)$ is the time-varying delay that satisfies $0 \leq \tau_{i}(t) \leq \tau_{\max }, U(l)$ is an external input (bias) vector to neurons, $f(x(t))=\left(f_{1}\left(x_{1}(t)\right), \cdots, f_{n}\left(x_{n}(t)\right)\right)^{T}$ and $f(x(t-\tau(t)))=\left(f_{1}\left(x_{1}\left(t-\tau_{1}(t)\right)\right), \cdots, f_{n}\left(x_{n}\left(t-\tau_{n}(t)\right)\right)\right)^{T}$ are vector-valued activation functions.

We shall consider two classes of activation functions for network (1). The first class of activation functions we consider includes sigmoid functions. It consists of all bounded continuous functions. Formally, we define the set

$$
\mathcal{B}=\left\{f(\cdot)\left|f_{i} \in C(\mathbb{R}, \mathbb{R}), \exists \kappa_{i}>0,\right| f_{i}\left(x_{i}\right) \mid \leq \kappa_{i}, \forall x_{i} \in \mathbb{R}, i=1,2, \cdots, n\right\} .
$$

The constants $\kappa_{i}, i=1,2, \cdots, n$, of the bounded activation functions will be called saturation constants. Note that for this class of activation functions, it is not required that they be monotone and $f(0)=0$. The second class of activation functions is of Lurie type, which may be unbounded and includes Lipschitz functions. It is defined by

$$
\Lambda=\left\{f(\cdot) \mid \exists \kappa_{i}>0,0 \leq x_{i} f_{i}\left(x_{i}\right) \leq \kappa_{i} x_{i}^{2}, \forall x_{i} \in \mathbb{R}, i=1,2, \cdots, n\right\} .
$$

The constants $\kappa_{i}, i=1,2, \cdots, n$, of Lurie-type functions will be called Lurie constants, and denoted $K=\operatorname{diag}\left(\kappa_{1}, \cdots, \kappa_{n}\right)$.

Let $C^{2,1}\left(\mathbb{R}^{n} \times \mathbb{R}_{+} \times \mathcal{M} ; \mathbb{R}^{+}\right)$denote the family of all nonnegative functions $V(x, t, l)$ on $\mathbb{R}^{n} \times \mathbb{R}_{+} \times \mathcal{M}$, which are continuously twice differentiable in $x$ and once in $t$. Define an operator $\mathcal{L} V$ [37] from $\mathbb{R}^{n} \times \mathbb{R}^{n} \times \mathbb{R}_{+} \times \mathcal{M}$ to $\mathbb{R}$ by

$$
\mathcal{L} V(x, y, t, l)=V_{t}(x, t, l)+V_{x}(x, t, l)[-D(l) x+A(l) f(x)+B(l) f(y)+U(l)]+\sum_{k=1}^{N} \gamma_{l k} V(x, t, k),
$$

where

$$
V_{t}(x, t, l)=\frac{\partial V(x, t, l)}{\partial t}, \quad V_{x}(x, t, l)=\left(\frac{\partial V(x, t, l)}{\partial x_{1}}, \cdots, \frac{\partial V(x, t, l)}{\partial x_{n}}\right) .
$$

Definition 2.1: Let $h: \mathbb{R} \rightarrow \mathbb{R}$ be a continuous function, $D^{+} h$ the upper Dini-derivative of $h$ is defined as

$$
D^{+} h(t)=\limsup _{\delta \rightarrow 0^{+}} \frac{h(t+\delta)-h(t)}{\delta} .
$$

Definition 2.2: A function $\alpha: R_{+} \rightarrow R_{+}$is said to be class of $\mathscr{K}$ if it is continuous and strictly increasing and satisfies $\alpha(0)=0$; it is of class $\mathscr{K}_{\infty}$ if in addition $\alpha(s) \rightarrow \infty$ as $s \rightarrow \infty$.

Definition 2.3: The trajectory of network (1) is said to be uniformly bounded in mean-square sense, if for any $\mathcal{H}>0$, there exists a constant $\mathcal{K}=\mathcal{K}(\mathcal{H})>0$ such that $E|x(t ; \varphi)|^{2}<\mathcal{K}$ for all $\varphi \in C(\mathcal{H})$ and $t \geq 0$.

Definition 2.4: Let $c^{(1)}=\left(c_{i}^{(1)}, \cdots, c_{n}^{(1)}\right)$ and $c^{(2)}=\left(c_{i}^{(2)}, \cdots, c_{n}^{(2)}\right)$ be real vector with $c^{(1)} \leq c^{(2)}$. If there exist radially unbounded and positive definite functions $V_{i}(x, l), i=1, \cdots, n, l \in \mathcal{M}$, functionals $\mathcal{K}_{i} \in \Pi, \chi \in \mathscr{K}_{\infty}$, positive constants $\beta_{i}$ and $\lambda$, such that for any solution $x(t)=x(t ; \varphi)$ of network $(1), t \geq 0$, implies

$$
c_{i}^{(1)}\left|x_{i}\right|^{2} \leq V_{i}(x, l) \leq c_{i}^{(2)}\left|x_{i}\right|^{2}, i=1, \cdots, n, l \in \mathcal{M}
$$

and

$$
\chi\left(E V_{i}(x(t), r(t))\right)-\beta_{i} \leq \mathcal{K}_{i}(\varphi) \exp \{-\lambda t\}
$$

then the trajectory of network (1) is said to be globally exponentially attractive with respect to $\left(V_{1}, \cdots, V_{n}\right)$ in mean square sense, and the compact set $\Omega:=\cap_{i=1}^{n} \cup_{l \in \mathcal{M}}\left\{x \in R^{n} \mid V_{i}(x, l) \leq \chi^{-1}\left(\beta_{i}\right)\right\}$ is called to be a globally exponentially attractive set (GEA) of network (1) in mean square sense.

Definition 2.5: The trajectory of network (1) is called mean-square globally exponentially stable in Lagrange sense, if it is both uniformly bounded in mean-square sense and globally exponentially attractive in mean-square sense.

Lemma 2.1: ([38]) If $A \in Z^{n \times n}$, then the following statements are equivalent:

(1) $A$ is a nonsingular M-matrix.

(2) $A$ is semipositive; that is, there exists $x \gg 0$ in $R^{n}$ such that $A x \gg 0$.

(3) $A^{-1}$ exists and its elements are all nonnegative. 


\section{Lagrange stability}

\subsection{Two important lemmas}

In this section, we establish two important Lemmas, which will play important role in the proofs of the main results.

Lemma 3.1. Assume that $x(t) \in C\left(\left[-\tau_{\max },+\infty\right], \mathbb{R}^{n}\right)$ is a real-valued $\mathcal{F}_{t}$-adapted process. If there exist $V \in C\left(\mathbb{R}^{n} \times\right.$ $\mathcal{M}, \mathbb{R})$, and constants $\alpha>0, \beta \geq 0, \forall t \geq 0$ such that

$$
D^{+} E V(x(t), r(t)) \leq-\alpha E V(x(t), r(t))+\beta \sqrt{E V(x(t), r(t))} .
$$

Then

$$
\sqrt{E V(x(t), r(t))}-\beta / \alpha \leq(\sqrt{E V(x(0), r(0))}-\beta / \alpha) \exp \{-\alpha t / 2\}, t \geq 0 .
$$

Proof: Let $W(t)=\sqrt{E V(x(t), r(t))}$, we have

$$
2 W(t) D^{+} W(t) \leq-\alpha W^{2}(t)+\beta W(t),
$$

which equals to

$$
D^{+} W(t) \leq-(\alpha / 2) W(t)+\beta / 2 .
$$

It follows from the Lemma 2.1 in [3], that

$$
W(t) \leq(W(0)-\beta / \alpha) \exp \{-\alpha t / 2\}+\beta / \alpha .
$$

Inequality (4) can be rewritten as

$$
\sqrt{E V(x(t), r(t))}-\beta / \alpha \leq(\sqrt{E V(x(0), r(0))}-\beta / \alpha) \exp \{-\alpha t / 2\} .
$$

The proof is completed.

Lemma 3.2. Assume that $x(t) \in C\left(\left[-\tau_{\max },+\infty\right], \mathbb{R}^{n}\right)$ is a real-valued $\mathcal{F}_{t}$-adapted process. Let $P=\operatorname{diag}\left\{p_{1}, p_{2}, \cdots, p_{n}\right\}$, $Q=\left(q_{i j}\right)_{n \times n}$, where $p_{i}>0, i=1,2, \cdots, n$. Assume that there exist $V_{i} \in C\left(\mathbb{R}^{n} \times \mathcal{M}, \mathbb{R}_{+}\right)(i=1,2, \ldots, n)$ such that $\forall t \geq 0$, the following condition holds:

$$
D^{+} E V_{i}(x(t), r(t)) \leq-p_{i} E V_{i}(x(t), r(t))+\sum_{j=1}^{n} q_{i j} \sup _{-\tau_{\max } \leq \theta \leq 0} \sqrt{E V_{j}(x(t+\theta), r(t+\theta))} \sqrt{E V_{i}(x(t), r(t))}+\beta_{i}, \beta_{i} \geq 0,
$$

if $p_{i}>\sum_{j=1}^{n} q_{i j}$, then for any $i=1,2, \ldots n$

$$
E V_{i}(x(t), r(t)) \leq E V(0) \exp \{-\lambda t\}+\gamma, t \geq 0,
$$

where

$$
\begin{gathered}
E V(0)=\sum_{i=1}^{n} E V_{i}(x(0), r(0)), \quad \gamma=\max _{1 \leq i \leq n}\left(p_{i}-\sum_{j=1^{n}} q_{i j}\right)^{-1} \beta_{i}, \\
\lambda=\min _{1 \leq i \leq n} \sup \left\{\lambda_{i}>0, p_{i}-\sum_{j=1}^{n} q_{i j} \exp \left\{\lambda_{i} \tau_{\max }\right\}-\lambda_{i}>0\right\} .
\end{gathered}
$$

Proof: In order to prove (6), it is just need to prove $\forall \tilde{\lambda} \in(0, \lambda)$

$$
E V_{i}(x(t), r(t)) \leq E V(0) \exp \{-\tilde{\lambda} t\}+\gamma .
$$

Let

$$
W_{i}(t)=E V_{i}(x(t), r(t)), \quad H(t)=E V(0) \exp \{-\tilde{\lambda} t\}+\gamma .
$$


Hence, it is just need to prove $\forall i=1,2, \ldots n$

$$
W_{i}(t) \leq H(t), \forall t \geq 0 .
$$

If (7) is not true, then there exist $\bar{i} \in(1,2, \ldots n)$ and $t^{*} \in[0, \infty)$ such that

$$
\begin{aligned}
& W_{\bar{i}}\left(t^{*}\right)=H\left(t^{*}\right), \\
& \mathcal{D}^{+} W_{\bar{i}}\left(t^{*}\right) \geq \mathcal{D}^{+} H\left(t^{*}\right), \\
& W_{i}(t) \leq H(t), 0 \leq t \leq t^{*}, i=1,2, \cdots, n .
\end{aligned}
$$

On the other hand, from the definition of $W_{i}(t),(5)$, and (9), we can obtain

$$
\begin{aligned}
& D^{+} W_{\bar{i}}\left(t^{*}\right)=D^{+} E V_{\bar{i}}\left(x\left(t^{*}\right), r\left(t^{*}\right)\right) \\
& \leq-p_{\bar{i}} E V_{\bar{i}}\left(x\left(t^{*}\right), r\left(t^{*}\right)\right)+\sum_{j=1}^{n} q_{\bar{i} j} \sup _{-\tau_{\max } \leq \theta \leq 0} \sqrt{E V_{j}\left(x\left(t^{*}+\theta\right), r\left(t^{*}+\theta\right)\right) E V_{\bar{i}}\left(x\left(t^{*}\right), r\left(t^{*}\right)\right)}+\beta_{\bar{i}} \\
& \leq \sum_{j=1}^{n} q_{\bar{i} j} \sqrt{\left(E V(0) \exp \left\{-\tilde{\lambda} t^{*}\right\}+\gamma\right)\left(E V(0) \exp \left\{-\tilde{\lambda}\left(t^{*}-\tau_{\max }\right)\right\}+\gamma\right)}-p_{\bar{i}}\left(E V(0) \exp \left\{-\tilde{\lambda} t^{*}\right\}+\gamma\right)+\beta_{\bar{i}} \\
& \leq\left(-p_{\bar{i}}+\sum_{j=1}^{n} q_{\bar{i} j} \exp \left\{\tilde{\lambda} \tau_{\max }\right\}\right) E V(0) \exp \left\{-\tilde{\lambda} t^{*}\right\}-p_{\bar{i}} \gamma+\sum_{j=1}^{n} q_{\bar{i} j} \gamma+\beta_{\bar{i}} .
\end{aligned}
$$

It follows from the definition of $\lambda$ and $\gamma$, it is easy to estimate that

$$
\begin{array}{r}
-p_{\bar{i}} \gamma+\sum_{j=1}^{n} q_{\bar{i} j} \gamma+\beta_{\bar{i}}<0, \\
-p_{\bar{i}}+\sum_{j=1}^{n} q_{\bar{i} j} \exp \left\{-\tilde{\lambda} t^{*}\right\}<-\tilde{\lambda} .
\end{array}
$$

Substituting (13), (14) into (12) yields

$$
D^{+} W_{\bar{i}}\left(t^{*}\right)<-\tilde{\lambda} E V(0) \exp \left\{-\tilde{\lambda} t^{*}\right\}=D^{+} H\left(t^{*}\right) .
$$

This contradict the inequality in (11). Hence, (8) holds. Letting $\tilde{\lambda} \rightarrow \lambda$, (06) holds.

Remark 3.1. 1. If $\beta_{i}=0$, the inequality in Lemma 3.2 degenerates into

$$
D^{+} E V_{i}(x(t), r(t)) \leq-p_{i} E V_{i}(x(t), r(t))+\sum_{j=1}^{n} q_{i j} \sup _{-\tau_{\max } \leq \theta \leq 0} \sqrt{E V_{j}(x(t+\theta), r(t+\theta))} \sqrt{E V_{i}(x(t), r(t))}
$$

which is present in [6]. Therefor, Lemma 3.2 generalized the Lemma 1 in [6].

2. When using scalar Lyapunov function, and $\beta_{i}=0$, the inequality (5) becomes

$$
D^{+} E V(x(t), r(t)) \leq-p E V(x(t), r(t))+q \sup _{-\tau_{\max } \leq \theta \leq 0} \sqrt{E V(x(t+\theta), r(t+\theta))} \sqrt{E V(x(t), r(t))} .
$$

Let $W(t)=\sqrt{E V(x(t), r(t))}$, the inequality above-mentioned can be rewrite as

$$
D^{+} W(t) \leq-p W(t)+q \sup _{-\tau_{\max } \leq \theta \leq 0} W(t+\theta)
$$

where $p>q>0$. This is the well-known Halanay inequality [39]. Hence, Lemma 3.2 is a generalization of the classical Halanay inequality. 


\subsection{Bounded feedback functions}

In this section, we will consider the bounded activation functions $f(\cdot) \in \mathcal{B}$.

Theorem 3.1. Assume that $f(\cdot) \in \mathcal{B}$. If there exists positive diagonal matrices $Q(l)=\operatorname{diag}\left\{q_{1}, \cdots, q_{n}\right\}, l \in \mathcal{M}$, such that $\alpha_{i}>0$, then network (1) is mean-square globally exponentially stable in Lagrange sense. Moreover, the compact set $\Theta_{1}=\bigcup_{l \in \mathcal{M}}\left\{x \in \mathbb{R}^{n} \mid, q_{i}(l) x_{i}^{2}(t) \leq\left(\beta_{i} / \alpha_{i}\right)^{2}\right\}$ is GEA set of $(1)$ in mean-square sense, where

$$
\alpha_{i}=\min _{1 \leq l \leq N}\left(2 d_{i}(l) q_{i}(l)-\sum_{k=1}^{N} \gamma_{l k} q_{i}(k)\right) \min _{1 \leq l \leq n} q_{i}(l)^{-1}, \quad \beta_{i}=2 \max _{1 \leq l \leq N} q_{i}(l)\left(\sum_{j=1}^{n}\left(\left|a_{i j}(l)\right|+\left|b_{i j}(l)\right|\right) \kappa_{j}+\left|u_{i}(l)\right|\right) \max _{1 \leq l \leq N} \sqrt{q_{i}(l)^{-1}} .
$$

Proof: Let $x_{i}(t)=x_{i}(t, \varphi), x_{i}^{\tau}(t)=x_{i}\left(t-\tau_{i}(t), \varphi\right)$ for simplicity. Consider the radially unbounded and positive definite Lyapunov function: $V_{i}(x(t), l)=q_{i}(l) x_{i}^{2}(t)$, from the definition of $\mathcal{L} V$ [37], we have

$$
\begin{aligned}
\mathcal{L} V_{i}\left(x(t), x^{\tau}(t), l\right) & =2 q_{i}(l) x_{i}(t)\left(-d_{i}(l) x_{i}(t)+\sum_{j=1}^{n}\left(a_{i j}(l) f_{j}\left(x_{j}(t)\right)+b_{i j}(l) f_{j}\left(x_{j}^{\tau}(t)\right)\right)+u_{i}(l)\right)+\sum_{k=1}^{N} \gamma_{l k} q_{i}(k) x_{i}^{2}(t) \\
& \leq\left(-2 d_{i}(l)+\sum_{k=1}^{N} \gamma_{l k} q_{i}(k) q_{i}(l)^{-1}\right)\left(q_{i}(l) x_{i}^{2}(t)\right)+2 \sum_{j=1}^{n} q_{i}(l)\left(\left(\left|a_{i j}(l)\right|+\left|b_{i j}(l)\right|\right) \kappa_{j}+\left|u_{i}(l)\right|\right)\left|x_{i}(t)\right| \\
& \leq \min _{1 \leq l \leq N}\left(-2 d_{i}(l) q_{i}(l)+\sum_{k=1}^{N} \gamma_{l k} q_{i}(k)\right) \min _{1 \leq l \leq N} q_{i}(l)^{-1} \max _{1 \leq l \leq N} q_{i}(l) x_{i}^{2}(t)+\beta_{i} \sqrt{\min _{1 \leq l \leq N} q_{i}(l) x_{i}^{2}(t)} \\
& =-\alpha_{i} \max _{1 \leq l \leq N} V_{i}(x(t), l)+\beta_{i} \sqrt{\min _{1 \leq l \leq N} V_{i}(x(t), l)} .
\end{aligned}
$$

By using the generalized Itô formula [37] and the definition of Dini-derivative, we have

$$
D^{+} E V_{i}(x(t), r(t)) \leq-\alpha_{i} E V_{i}(x(t), r(t))+\beta_{i} E \sqrt{V_{i}(x(t), r(t))} .
$$

The well-known Hölder inequality, yields

$$
D^{+} E V_{i}(x(t), r(t)) \leq-\alpha_{i} E V_{i}(x(t), r(t))+\beta_{i} \sqrt{E V_{i}(x(t), r(t))} .
$$

According to the Lemma 3.1, we have

$$
\sqrt{E V_{i}(x(t), r(t))}-\beta_{i} / \alpha_{i} \leq\left(\sqrt{E V_{i}(x(0), r(0))}-\beta_{i} / \alpha_{i}\right) \exp \left\{-\alpha_{i} t / 2\right\} .
$$

This immediately implies the uniform boundedness of the solutions of $(1)$. Let $\mathcal{K}_{i}(\varphi)=\sqrt{E V_{i}(x(0), r(0))}=\sqrt{q_{i}(r(0)) \varphi^{2}(0)}$. Then $\mathcal{K}_{i} \in \Pi$, and (15) implies that for all $t \geq 0$,

$$
\sqrt{E V_{i}(x(t), r(t))}-\beta_{i} / \alpha_{i} \leq \mathcal{K}_{i}(\varphi) \exp \left\{-\alpha_{i} t / 2\right\}
$$

By Definition 2.4 and Definition 2.5, network (1) is mean-square globally exponentially attractive and $\Theta_{1}$ is a GEA set. This proves the GES (in Lagrange sense) of (1). The proof is completed.

When $D(l), A(l), B(l)$ are independent on $l$, then the network (1) becomes the deterministic system presented in Liao et al. [3], i.e.,

$$
\dot{x}(t)=-D x(t)+A f(x(t))+B f(x(t-\tau(t)))+U,
$$

we can obtain following Corollary.

Corollary 3.1. Assume that $f(\cdot) \in \mathcal{B}$. If $D=\operatorname{diag}\left\{d_{1}, \cdots, d_{n}\right\}$ is a positive diagonal matrix, then network (16) is globally exponentially stable in Lagrange sense. Moreover, the compact set $\Theta_{2}$ is GEA set of (16), where

$$
\Theta_{2}=\left\{x \in \mathbb{R}^{n} \mid x_{i}^{2} \leq\left(\frac{\beta_{i}}{d_{i}}\right)^{2}, i=1,2, \cdots, n\right\}, \quad \beta_{i}=\left(\sum_{j=1}^{n}\left(\left|a_{i j}\right|+\mid b_{i j}\right)\left|\kappa_{j}+\right| u_{i} \mid\right) .
$$


Remark 3.2. If $f(\cdot) \in \mathcal{B}$, Liao et al provided the GEA set $\Omega_{1}$ in [3] for the network (16) as follows

$$
\Omega_{1}=\left\{x \in \mathbb{R}^{n} \mid \sum_{i=1}^{n} x_{i}^{2} \leq \frac{\sum_{i=1}^{n}\left(\beta_{i}\right)^{2} / \varepsilon_{i}}{4 \min _{1 \leq i \leq n}\left(d_{i}-\varepsilon_{i}\right)}, 0<\varepsilon_{i}<d_{i}\right\} .
$$

Noting that $d_{i}^{2} \geq 4 \varepsilon_{i}\left(d_{i}-\varepsilon_{i}\right), 0<\varepsilon_{i}<d_{i}$, simple computation shows that

$$
\sum_{i=1}^{n}\left(\frac{\beta_{i}}{d_{i}}\right)^{2} \leq \sum_{i=1}^{n} \frac{\left(\beta_{i}\right)^{2} / \varepsilon_{i}}{4\left(d_{i}-\varepsilon_{i}\right)} \leq \frac{\sum_{i=1}^{n}\left(\beta_{i}\right)^{2} / \varepsilon_{i}}{4 \min _{1 \leq i \leq n}\left(d_{i}-\varepsilon_{i}\right)}
$$

This implies $\Theta_{2} \subset \Omega_{1}$. As a result, we provided a more accurate estimate for the GEA set than that of Liao et al. in [3].

\subsection{Lurie-type feedback functions}

In this section, we assume the activation function $f(\cdot) \in \Lambda$.

Theorem 3.2. Assume that $f(\cdot) \in \Lambda$. If there exist positive diagonal matrices $S(l)=\operatorname{diag}\left\{s_{1}(l), s_{2}(l), \cdots, s_{n}(l)\right\}$, $l \in \mathcal{M}$, such that $P-Q$ is a dominant principal diagonal matrix, where $P=\operatorname{diag}\left\{p_{1}, p_{2}, \cdots, p_{n}\right\}$ is a positive diagonal matrix, $Q=\left(q_{i j}\right)_{n \times n}$ with

$$
\begin{gathered}
p_{i}=\min _{1 \leq l \leq N}\left(\left(2 d_{i}(l)-2 \bar{a}_{i i}(l) \kappa_{i}\right) s_{i}^{2}(l)-\sum_{k=1}^{N} \gamma_{l k} s_{i}^{2}(k)\right) \min _{\leq l \leq n} s_{i}^{-2}(l), \\
q_{i j}=\left\{\begin{array}{l}
\max _{1 \leq l \leq N}\left(2 s_{i}^{2}(l)\left(\left|a_{i j}(l)\right|+\left|b_{i j}(l)\right|\right) \kappa_{j}\right) \max _{\leq l \leq n} s_{i}^{-1}(l) \max _{\leq l \leq n} s_{j}^{-1}(l), j \neq i, \\
\max _{1 \leq l \leq N} 2 s_{i}^{2}(l)\left|b_{i i}(l)\right| \kappa_{i} \max _{\leq l \leq n} s_{i}^{-2}(l),
\end{array}, i .\right.
\end{gathered}
$$

Then network (1) is mean-square globally exponentially stable in Lagrange sense. Moreover, the compact set $\Theta_{3}=$ $\bigcup_{l \in \mathcal{M}}\left\{x \in \mathbb{R}^{n} \mid x_{i}^{2} \leq \gamma(\varepsilon) s_{i}^{-2}(l), s_{i}(l)>0, i=1,2, \cdots, n\right\} i s$ a GEA set of $(1)$ in mean-square sense, where

$$
\gamma(\varepsilon)=\max _{1 \leq i \leq n}\left(p_{i}\left(\varepsilon_{i}\right)-\sum_{j=1}^{n} q_{i j}\right)^{-1} \beta_{i}\left(\varepsilon_{i}\right), \quad p_{i}\left(\varepsilon_{i}\right)=\min _{1 \leq l \leq N}\left(\left(2 d_{i}(l)-2 \bar{a}_{i i}(l) \kappa_{i}-\varepsilon_{i}\right) s_{i}^{2}(l)-\sum_{k=1}^{N} \gamma_{l k} s_{i}^{2}(k)\right) \min _{1 \leq l \leq N} s_{i}^{-2}(l),
$$

$\beta_{i}\left(\varepsilon_{i}\right)=\varepsilon_{i}^{-1} \max _{1 \leq l \leq N} s_{i}^{2}(l) u_{i}^{2}(l), \varepsilon_{i}>0$ are any sufficient small constants satisfying $p_{i}\left(\varepsilon_{i}\right)>\sum_{j=1}^{n} q_{i j}, i=1,2, \cdots, n$.

Proof: Let $x_{i}(t)=x_{i}(t, \varphi), x_{i}^{\tau}(t)=x_{i}\left(t-\tau_{i}(t), \varphi\right)$ for simplicity. Applying Itô formula to $V_{i}(x(t), l)=s_{i}^{2}(l) x_{i}^{2}(t)$, yields

$$
\begin{aligned}
\mathcal{L} V_{i}(x(t), l)= & 2 s_{i}^{2}(l) x_{i}(t)\left(-d_{i}(l) x_{i}+\sum_{j=1}^{n}\left(a_{i j}(l) f_{j}\left(x_{j}(t)\right)+b_{i j}(l) f_{j}\left(x_{j}^{\tau}(t)\right)\right)+u_{i}(l)\right)+\sum_{k=1}^{N} \gamma_{l k} s_{i}^{2}(k) x_{i}^{2}(t) \\
\leq & -\left(\left(2 d_{i}(l)-2 \bar{a}_{i i}(l) \kappa_{i}-\varepsilon_{i}\right) s_{i}^{2}(l)-\sum_{k=1}^{N} \gamma_{l k} s_{i}^{2}(k)\right) \min _{1 \leq l \leq N} s_{i}^{-2}(l) \max _{1 \leq l \leq N} s_{i}^{2}(l) x_{i}^{2}(t) \\
& +2 \sum_{j=1, j \neq i}^{n} s_{i}^{2}(l)\left|a_{i j}(l)\right| \kappa_{j} \max _{1 \leq l \leq N} s_{i}^{-1}(l) \max _{1 \leq l \leq N} s_{j}^{-1}(l) \min _{1 \leq l \leq N} s_{i}(l)\left|x_{i}(t)\right| \min _{1 \leq l \leq N} s_{j}(l)\left|x_{j}(t)\right| \\
& +2 \sum_{j=1}^{n} s_{i}^{2}(l)\left|b_{i j}(l)\right| \kappa_{j} \max _{1 \leq l \leq N} s_{i}^{-1}(l) \max _{1 \leq l \leq N} s_{j}^{-1}(l) \min _{1 \leq l \leq N} s_{i}(l)\left|x_{i}(t)\right| \min _{1 \leq l \leq N} s_{j}(l)\left|x_{j}^{\tau}(t)\right|+s_{i}^{2}(l) u_{i}^{2}(l) \varepsilon_{i}^{-1} \\
\leq & -p_{i}\left(\varepsilon_{i}\right) \max _{1 \leq l \leq N} V_{i}(x(t), l)+\sum_{j=1}^{n} q_{i j} \sup _{-\tau_{\max } \leq \theta \leq 0} \sqrt{\min _{1 \leq l \leq N} V_{j}(x(t+\theta), l)} \sqrt{\min _{1 \leq l \leq N} V_{i}(x(t), l)}+\beta_{i}\left(\varepsilon_{i}\right) .
\end{aligned}
$$


Hence, by using the generalized Itô formula [37] and Hölder inequality, yields

$$
D^{+} E V_{i}(x(t), l) \leq-p_{i}\left(\varepsilon_{i}\right) E V_{i}(x(t), l)+\sum_{j=1}^{n} q_{i j} \sup _{-\tau_{\max } \leq \theta \leq 0} \sqrt{E V_{j}(x(t+\theta), l)} \sqrt{E V_{i}(x(t), l)}+\beta_{i}\left(\varepsilon_{i}\right) .
$$

Noting that $P-Q$ is a dominant principal diagonal matrix, by the continuity, there exist $\varepsilon_{i}>0, i=1,2, \cdots, n$, such that $P(\varepsilon)-Q$ is still a dominant principal diagonal matrix. According to Lemma 3.2, for any $i=1,2, \cdots, n$

$$
E V_{i}(x(t), r(t)) \leq E V(0) \exp \{-\lambda t\}+\gamma(\varepsilon)
$$

where

$$
E V(0)=\sum_{i=1}^{n}\left\{E V_{i}(x(0), r(0))\right\}, \quad \lambda=\min _{1 \leq i \leq n} \sup \left\{\lambda>0, p_{i}\left(\varepsilon_{i}\right)-\sum_{l=1}^{n} q_{i j} \exp \left\{\lambda \tau_{\max }\right\}-\lambda>0\right\} .
$$

It implies the uniform boundedness of the solutions of (1). Let $\mathcal{K}(\varphi)=E \sum_{i=1}^{n}\left\{s_{i}^{2}(r(0)) x_{i}^{2}(0)\right\}=E \sum_{i=1}^{n}\left\{s_{i}^{2}(r(0)) \varphi_{i}^{2}(0)\right\}$. Then $\mathcal{K} \in \Pi$, and (17) implies

$$
E V_{i}(x(t), r(t))-\gamma(\varepsilon) \leq \mathcal{K}(\varphi) \exp \{-\lambda t\}, t \geq 0 .
$$

By Definition 2.4, and Definition 2.5 network (1) is mean-square globally exponentially attractive and $\Theta_{3}$ is a GEA set in mean-square sense.

Remark 3.3. The globally exponentially stable in Lagrange sense for deterministic DRNNs has been discussed in $[3,8]$, but the used LMI approach is not applicable for DRNNs with Markovian switching due to the existence of the Markovian switching mechanism. With the help of Lemma 3.2 and vector Lyapunov functions, the Lagrange stability of DRNNs with Markovian switching can be investigated.

Remark 3.4. When the network (1) degenerates to network (16), the main condition of Theorem 3.2 becomes $2 d_{i}-$ $2 a_{i i} \kappa_{i}>2\left|b_{i i}\right| \kappa_{i}+2 \sum_{i \neq j, j=1}^{n}\left(\left|\bar{a}_{i j}\right|+\left|b_{i j}\right|\right) \kappa_{j} s_{i} s_{j}^{-1}, i=1,2, \cdots, n$, which means $d_{i} s_{i}^{-1}>\sum_{j=1}^{n}\left(\left|\bar{a}_{i j}\right|+\left|b_{i j}\right|\right) \kappa_{j} s_{j}^{-1}, i=$ $1,2, \cdots, n$. Thus, $D-(|\bar{A}|+|B|) K$ is a nonsingular M- matrix by Lemma 2.1. Hence, we can obtain the following corollary.

Corollary 3.2. Assume that $f(\cdot) \in \Lambda$. If $D-(|\bar{A}|+|B|) K$ is a nonsingular $M$ - matrix, then network (16) is globally exponentially stable in Lagrange sense. Moreover, the compact set $\Theta_{4}=\left\{x \in \mathbb{R}^{n} \mid x_{i}^{2} \leq \gamma(\varepsilon) s_{i}^{-2}, i=1,2, \cdots, n\right\}$ is a GEA set of (16), where $\gamma(\varepsilon)=\max _{1 \leq i \leq n}\left(2 d_{i}-2 \sum_{j=1}^{n}\left(\left|\bar{a}_{i j}\right|+\left|b_{i j}\right|\right) \kappa_{j} s_{i} s_{j}^{-1}-\varepsilon_{i}\right)^{-1} \beta_{i}\left(\varepsilon_{i}\right), \quad \beta_{i}\left(\varepsilon_{i}\right)=s_{i}^{2} u_{i}^{2} \varepsilon_{i}^{-1}$ with $\varepsilon_{i}>0, i=1,2, \cdots, n, S=\operatorname{diag}\left\{s_{1}, \cdots, s_{n}\right\}$ satisfying $\left.2 d_{i}-\varepsilon_{i}-2 \sum_{j=1}^{n} s_{i} s_{j}^{-1}\left(\left|\bar{a}_{i j}\right|\right)+\left|b_{i j}\right|\right) \kappa_{j}>0$.

Remark 3.5. Although some Lagrange stability criteria for DRNNs have been derived by LMI approach in [8] and [17]-[19], the restrictive condition that the derivative value of time-varying dealy is less than one must be observed. With the help of M-matrix technique and vector Lyapunov function methods, sufficient conditions on Lagrange stability have been derived without imposing the restrictive condition.

Remark 3.6. In Corollary 3.2, if $B=0$, the network (16) becomes

$$
\dot{x}(t)=-D x(t)+A f(x(t))+U,
$$

and if $D-|\bar{A}| K$ is a nonsingular M-matrix, then the network (18) is globally exponentially stable in Lagrange sense. The assumption of Theorem 4.3 in [3] is $-d_{j} / \kappa_{j}+\bar{a}_{j j}+\sum_{i=1, i \neq j}^{n}\left|\bar{a}_{i j}\right|<0$, which implies that $D-|\bar{A}| K$ is a nonsingular M-matrix, not vice versa.

\section{Numerical simulations and comparisons}

In this section, we perform some numerical simulations to illustrate our results. 
Example 4.1. Consider the following delayed two neuron networks with Markovian switching

$$
\dot{x}(t)=-D(r(t)) x(t)+A(r(t)) f(x(t))+B(r(t)) f(x(t-\tau(t)))+U(r(t))
$$

where the generator of Markovian chain and other parameters are

$$
\begin{gathered}
\Gamma=\left(\begin{array}{cc}
-1 & 1 \\
1 & -1
\end{array}\right), D(1)=\left(\begin{array}{cc}
2.5 & 0 \\
0 & 2
\end{array}\right), D(2)=\left(\begin{array}{cc}
2 & 0 \\
0 & 2.5
\end{array}\right), A(1)=\left(\begin{array}{cc}
1.05 & -0.5 \\
0.1 & 0.6
\end{array}\right), A(2)=\left(\begin{array}{cc}
1 & 0.5 \\
-0.1 & 0.5
\end{array}\right), \\
B(1)=\left(\begin{array}{cc}
2 & 0.5 \\
0.45 & 0.9
\end{array}\right), B(2)=\left(\begin{array}{cc}
1.9 & -0.5 \\
0.5 & 0.8
\end{array}\right), U(1)=\left(\begin{array}{l}
1 \\
1
\end{array}\right), U(2)=\left(\begin{array}{l}
-1 \\
-1
\end{array}\right),
\end{gathered}
$$

and $f(x)=(|x+1|-|x-1|) / 2, \tau_{1}(t)=\tau_{2}(t)=1.2|\sin (t)|$. Choose $V_{i}(x(t), l)=q_{i}(l) x_{i}^{2}(t)$ with $q_{1}(1)=1, q_{1}(2)=$ $1.1, q_{2}(1)=q_{2}(2)=1$. Since $f(x)$ is bounded and $\kappa_{1}=\kappa_{1}=1$, it is easy to figure up $\alpha_{1}=4.0909, \alpha_{2}=4.0000$. According to Theorem 3.1, we conclude that the network (19) is mean-square globally exponentially stable in the Lagrange sense. Moreover, $\beta_{1}=10.2783 \beta_{2}=6.1000$. Hence, the set of the global exponential attraction $\Theta=\{x \in$ $\left.\mathbb{R}^{2} \mid x_{1}^{2} \leq 6.3126, x_{2}^{2} \leq 2.3256\right\} \cup\left\{x \in \mathbb{R}^{2} \mid x_{1}^{2} \leq 5.7387, x_{2}^{2} \leq 2.3256\right\}$. The Figure 1 (a), (b), (c) with 5 random initial values verify the feasibility of our results.

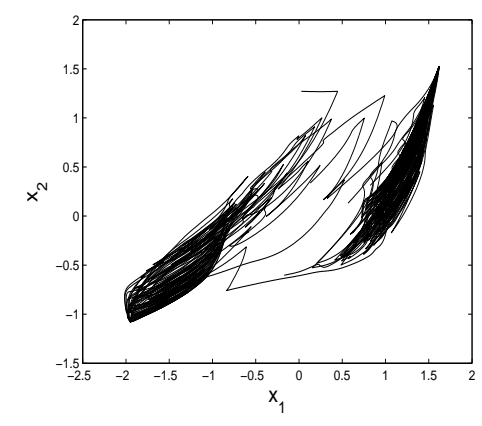

(a)

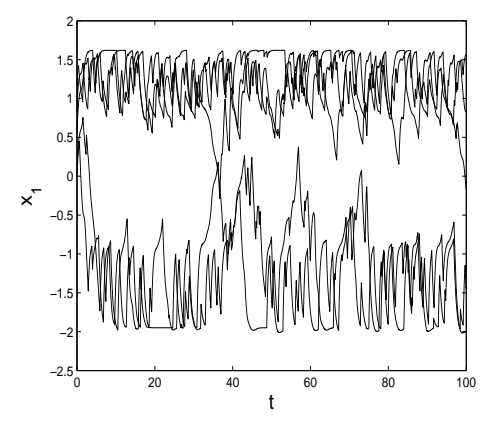

(b)

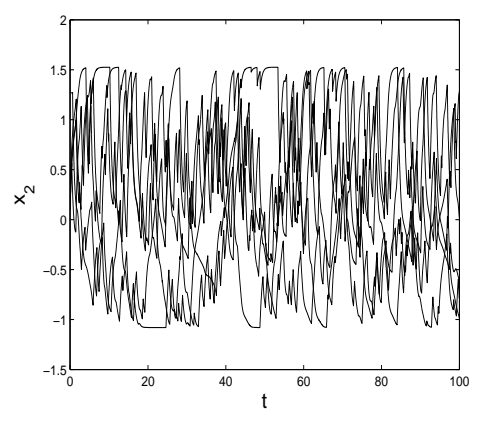

(c)

Figure 1: Transient behaviors of $x(t)$ of network(19).

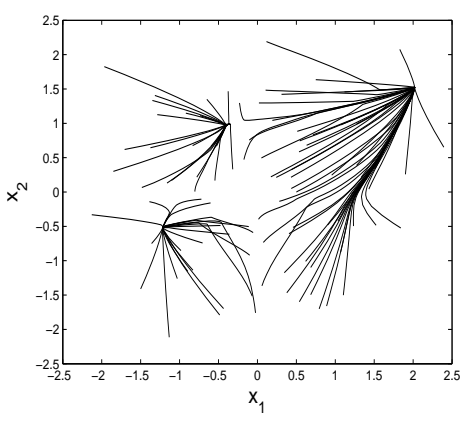

(a)

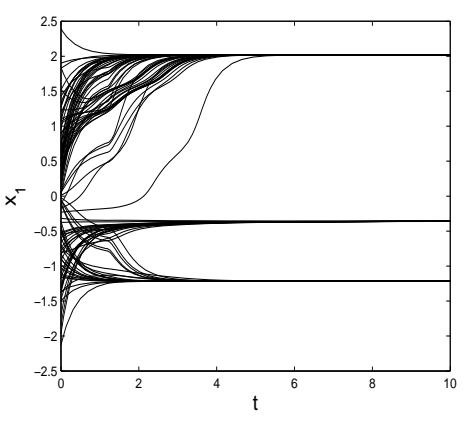

(b)

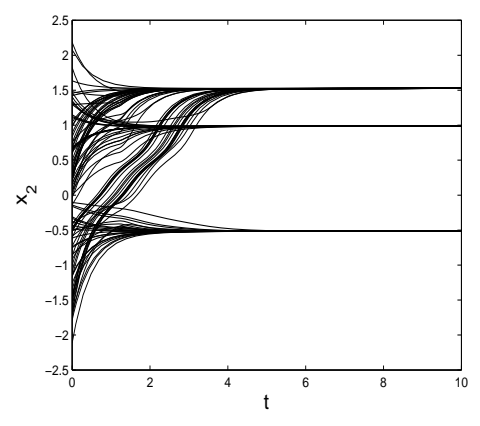

(c)

Figure 2: Transient behaviors of $x(t)$ of network(20).

Remark 4.1. Consider the first subsystem in Example 4.1

$$
\dot{x}(t)=-D(1) x(t)+A(1) f(x(t))+B(1) f(x(t-\tau(t)))+U(1) .
$$


Taking the activation function $f_{1}(x)=f_{2}(x)=\tanh (5.1 x), \tau_{1}(t)=\tau_{2}(t)=1.2$. According to Corollary 3.1, the network (20) is globally exponentially stable in Lagrange sense. Since $d_{1}=2.5, d_{2}=2, \beta_{1}=5.05, \beta_{2}=3.05$, we can easily get the globally exponentially attractive set $\Theta_{2}=\left\{x \in \mathbb{R}^{2} \mid x_{1}^{2} \leq 4.0804, x_{2}^{2} \leq 2.3256\right\}$. Noting that the globally exponentially attractive set provided by Liao et al. in [3] is $\Omega_{1}=\left\{x \in \mathbb{R}^{2} \mid x_{1}^{2}+x_{2}^{2} \leq \sum_{i=1}^{2} \beta_{i}^{2} \varepsilon_{i}^{-1} /\left(4 \min _{1 \leq i \leq 2}\left(d_{i}-\varepsilon_{i}\right)\right), 0<\right.$ $\left.\varepsilon_{i}<d_{i}\right\}$. Simple computation shows that $\Theta_{2} \subset \Omega_{1}$ for any $0<\varepsilon_{i}<d_{i}$. Hence, we provided a better estimation for the region of global attraction. The Figure 2 (a), (b), (c) with 100 random initial values verify the feasibility of our results.

Example 4.2. Consider the following delayed two-neuron networks with Markovian switching parameters

$$
\dot{x}(t)=-D(r(t)) x(t)+A(r(t)) f(x(t))+B(r(t)) f(x(t-\tau(t)))+U(r(t)),
$$

where the generator of Markovian chain and other parameters are

$$
\begin{gathered}
\Gamma=\left(\begin{array}{cc}
-1 & 1 \\
\frac{1}{2} & -\frac{1}{2}
\end{array}\right), D(1)=\left(\begin{array}{cc}
1.4 & 0 \\
0 & 1.5
\end{array}\right), D(2)=\left(\begin{array}{cc}
1.5 & 0 \\
0 & 1.6
\end{array}\right), A(1)=\left(\begin{array}{cc}
0 & 0.5 \\
0.5 & 0
\end{array}\right) \\
A(2)=\left(\begin{array}{cc}
-0.1 & 0.4 \\
0.6 & 0.1
\end{array}\right), B(1)=\left(\begin{array}{cc}
0 & 0.5 \\
0.5 & 0
\end{array}\right), B(2)=\left(\begin{array}{cc}
0 & 0.6 \\
0.2 & 0
\end{array}\right), U(1)=\left(\begin{array}{l}
1 \\
1
\end{array}\right), U(2)=\left(\begin{array}{l}
0 \\
1
\end{array}\right),
\end{gathered}
$$

$f_{1}\left(x_{1}\right)=\left|x_{1}\right| \tanh \left(x_{1}\right), f_{2}\left(x_{2}\right)=\tanh \left(x_{2}\right), x_{1}, x_{2} \in \mathbb{R}, \tau_{1}(t)=\tau_{2}(t)=1.2|\sin (t)|$. Then $f \in \Lambda, \kappa_{1}=\kappa_{2}=1$. Let $V_{i}(x(t), l)=s_{i}^{2}(l) x_{i}^{2}(t)$ with $s_{1}(1)=\sqrt{1.1}, s_{1}(2)=1, s_{2}(1)=\sqrt{1.1}, s_{2}(2)=1, \varepsilon_{1}=0.45, \varepsilon_{2}=0.45$. We can easily derived

$$
Q=\left(\begin{array}{ll}
0 & 2 \\
2 & 0
\end{array}\right), P=\left(\begin{array}{cc}
2.89 & 0 \\
0 & 2.9
\end{array}\right), P(\varepsilon)=\left(\begin{array}{cc}
2.44 & 0 \\
0 & 2.45
\end{array}\right), \beta_{1}\left(\varepsilon_{1}\right)=1.1 / 0.45, \beta_{2}\left(\varepsilon_{2}\right)=1 / 0.45
$$

Since $P-Q$ is a dominant principal diagonal matrix, according to Theorem 3.2, the network (21) is mean-square globally exponentially stable in Lagrange sense. And $\gamma(\varepsilon)=\max _{1 \leq i \leq 2}\left(p_{i}\left(\varepsilon_{i}\right)-\sum_{j=1}^{2} q_{i j}\right)^{-1} \beta_{i}\left(\varepsilon_{i}\right)=5.5556$, this implies the attraction

$$
\Theta=\left\{x \in \mathbb{R}^{2} \mid\left(x_{1}^{2} \leq 5.0505, x_{2}^{2} \leq 5.0505\right)\right\} \cup\left\{x \in \mathbb{R}^{2} \mid\left(x_{1}^{2} \leq 5.5556, x_{2}^{2} \leq 5.5556\right)\right\}
$$

The Figure 3 (a) verify the feasibility of our results.

Remark 4.2. In Example 4.2, when (21) becomes the deterministic networks

$$
\dot{x}(t)=-D x(t)+A f(x(t))+B f(x(t-\tau(t)))+U
$$

with $D=D(1), A=A(1), B=B(1), U=U(1), f(x)=|x| \tanh (x), \tau_{1}(t)=\tau_{2}(t)=1.2|\sin (t)|$, since $D-(|\bar{A}|+|B|) K$ is a M-matrix, according to the Corollary 3.2, the network (22) is globally exponentially stable in Lagrange sense. Noting that $(D-(|\bar{A}|+|B|) K)(1,1)^{T} \gg 0$, let $V_{i}(x)=x_{i}^{2}$, take $S=\operatorname{diag}\{1,1\}, \varepsilon_{1}=0.4, \varepsilon_{2}=0.5$, then by Corollary 3.2 , we can easily figure up $\beta_{1}\left(\varepsilon_{1}\right)=2.5, \beta_{2}\left(\varepsilon_{2}\right)=2, \gamma(\varepsilon)=6.2500$. This implies the attraction

$$
\Theta=\left\{x \in \mathbb{R}^{2} \mid\left(x_{1}^{2} \leq 6.2500, x_{2}^{2} \leq 6.2500\right)\right\} .
$$

The global exponential stability of the equilibrium point $(2.1266,2.0446)^{T}$ is demonstrated in Figure 3 (b), (c). Let the matrices

$$
Q^{(1)}=\left(\begin{array}{cc}
Q_{11}^{(1)} & Q_{12}^{(1)} \\
\left(Q_{12}^{(1)}\right)^{T} & Q_{22}^{(1)}
\end{array}\right)_{4 \times 4}, Q^{(2)}=\left(\begin{array}{cc}
Q_{11}^{(2)} & Q_{12}^{(2)} \\
\left(Q_{12}^{(2)}\right)^{T} & Q_{22}^{(2)}
\end{array}\right)_{4 \times 4}
$$

where $Q_{11}^{(1)}=\left(\bar{A}(1)+\bar{A}(1)^{T}\right) / 2+E_{2 \times 2}-\operatorname{diag}\left\{d_{1}(1) / \kappa_{1}, d_{2}(1) / \kappa_{2}\right\}, Q_{12}^{(1)}=Q_{12}^{(2)}=B(1) / 2, Q_{22}^{(1)}=Q_{22}^{(2)}=-E_{2 \times 2}, Q_{11}^{(2)}=$ $\left(\bar{A}(1)+\bar{A}(1)^{T}\right) / 2+E_{2 \times 2}, \kappa_{1}=\kappa_{2}=1$. Then $\lambda_{\min }\left\{Q^{(1)}\right\}=-1.4768<0, \lambda_{\max }\left\{Q^{(1)}\right\}=0.2522>0, \lambda_{\min }\left\{Q^{(2)}\right\}=$ $-1.1514<0, \lambda_{\max }\left\{Q^{(2)}\right\}=1.5963>0$. Hence, the matrices $Q^{(1)}, Q^{(2)}$ are indefinite matrices, these conditions of Theorem 4.1 and Theorem 4.2 in [3] are not satisfied. Therefore, it fails to conclude whether network (22) is globally exponentially stable in Lagrange sense. 


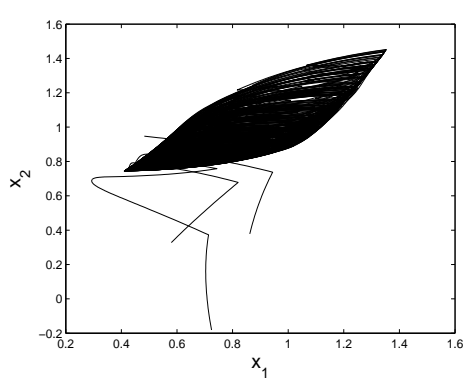

(a)

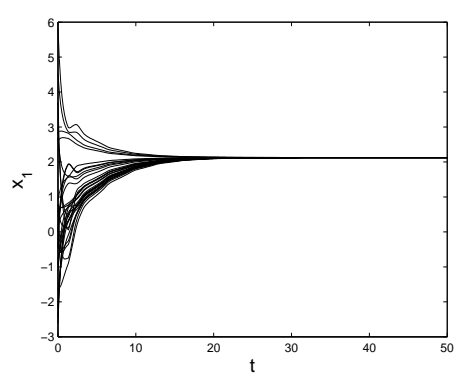

(b)

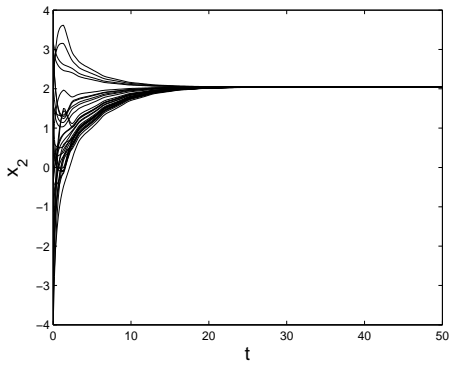

(c)

Figure 3: Transient behaviors of $x(t)$ with $\tau_{1}(t)=\tau_{2}(t)=1.2|\sin (t)|$ for the networks (21) and (22).

\section{Concluding remarks}

In this paper, we have investigated the mean-square global exponential stability in Lagrange sense for DRNNs with Markovian switching. By employing the vector Lyapunov function and M-matrix theory, two $\mathcal{L}$-operator differential inequalities have been established. With the help of the two $\mathcal{L}$-operator differential inequalities, some criteria for the Lagrange stability in mean-square sense of DRNNs with Markovian switching have been obtained. Finally, two numerical examples are provided to illustrate the efficiency of the derived results.

\section{Acknowledgements}

The project reported here is supported by the National Science Foundation of China (Grant Nos. 61304070, 11271146), the National Key Basic Research Program of China (973 Program) (2013CB228204) and the Fundamental Research Funds for the Central Universities of China. (Grant No.2015B19814).

\section{References}

[1] J. J. Hopfield, Neurons with graded response have collective computational properties like those of two-stateneurons, Proceedings of the National Academy of Sciences 81 (1984) 3088-3092.

[2] C. G. Huang, Y. G. He, H. N. Wang, Mean square exponential stability of stochastic recurrent neural networks with time-varying delays, Computers and Mathematics with Applications 56 (2008) 1773-1778.

[3] X. X. Liao, Q. Luo, Z. G. Zeng, Y. X. Guo, Global exponential stability in Lagrange sense for recurrent neural networks with time delays, Nonlinear Analysis: Real World Applications 9 (2008) 1535-1557.

[4] X. X. Liao, J. Wang, Global dissipativity of continuous-time recurrent neural networks with time delay, Physical Review E 68 (2003) 016118.

[5] Q. Luo, Z. G. Zeng, X. X. Liao, Global exponential stability in Lagrange sense for neutral type recurrent neural networks, Neurocomputing 74 (2011) 638-645.

[6] Y. Shen, J. Wang, Almost sure exponential stability of recurrent neural networks with Markovian switching, IEEE Transactions on Neural Networks 20 (5) (2009) 840-855.

[7] Y. H. Sun, J. D. Cao, pth moment expontial stability of stochastic recurrent neural networks with time-varying delays, Nonlinear Analysis: Real World Applications 8 (2007) 1171-1185.

[8] Z.W. Tu, J. G.Jian, K. Wang, Global exponential stability in Lagrange sense for recurrent neural networks with both time-varying delays and general activation functions via LMI approach, Nonlinear Analysis: Real World Applications 12 (2011) $2174-2182$.

[9] H. Wersing, W. Beyn, H. Ritter, Dynamical stability conditions for recurrent neural networks with unsaturating piecewise linear transfer functions, Neural Computation 13 (2001) 1811-1825.

[10] Z. Yi, K. K. Tan, Convergence Analysis of Recurrent Neural Networks, Kluwer Academic Publishers, Dordrecht, 2004.

[11] G. Zhang, Y. Shen, Q. Yin, J. Sun, Global exponential periodicity and stability of a class of memristor-based recurrent neural networks with multiple delays, Information Sciences 232 (2013) 386-396.

[12] G. D. Zhang, Y. Shen, C. J. Xu, Global exponential stability in a Lagrange sense for memristive recurrent neural networks with time-varying delays, Neurocomputing 149 (2015) 1330-1336.

[13] A. L. Wu, Z. Z. Zeng, X. S. Zhu, J. Zhang, Exponential synchronization of memristor-based recurrent neural networks with time delays, Neurocomputing 74 (2011) 3043-3050.

[14] A. L.Wu, Z. Zeng, Exponential synchronization of memristor-based recurrent neural networks with time delays, IEEE Transactions on Neural Networks and Learning Systems 23 (2012) 1919-1929.

[15] A. L. Wu, Z. Zeng, Dynamic behaviors of memristor-based recurrent neural networks with time-varying delays, Neural Networks 36 (2011) $1-10$.

[16] G. Seiler, J. A. Nossek, Winner-take-all cellular neural networks, IEEE Transaction on Circuits and Systems II 40 (1993) 184-190. 
[17] B. X. Wang, J. G. Jian, M. H. Jiang, Stability in Lagrange sense for Cohen-Grossberg neural networks with time-varying delays and finite distributed delays, Nonlinear Analysis:Hybrid Systems 4 (2010) 65-78.

[18] J. G. Jian, B. X. Wang, Global Lagrange stability for neutral-type Cohen-Grossberg BAM neural networks with mixed time-varying delays, Mathematics and Computers in Simulation 116 (2015) 1-25.

[19] X. H.Wang, M. H. Jiang, S. L. Fang, Stability analysis in Lagrange sense for a non-autonomous Cohen-Grossberg neural network with mixed delays, Nonlinear Analysis 70 (2009) 4294-4306.

[20] A. L.Wu, Z. G. Zeng, C. J. Fu, W. W. Shen, Global exponential stability in Lagrange sense for periodic neural networks with various activation functions, Neurocomputing 74 (2011) 831-837.

[21] X. X. Liao, Q. Luo, Z. G. Zeng, Positive invariant and global exponential attractive sets of neural networks with time-varying delays, Neurocomputing 71 (2008) 513-518.

[22] C. Briat, Linear Parameter-Varying and Time-Delay Systems, Berlin, Germany: Springer, 2015.

[23] K. Gu, V. Kharitonov, J. Chen, Stability of Time-Delay Systems, Boston, MA, USA:Birkhauser, 2003.

[24] L. R. Huang, X. R. Mao, Delay-dependent exponential stability of neutral stochastic delay systems, IEEE Transactions on Automatic Control 54 (2009) 147-152.

[25] S. Xu, Y. Chu, J. Lu, Y. Zou, Exponential dynamic output feedback controller design for stochastic neutral systems with distributed delays, IEEE Transactions on Systems Man and Cybernetics Part A-Systems and Humans 36 (2006) 540-548.

[26] A. Cheng, C. C. Lim, Markov modelling and parameterisation of genetic evolutionary test generations, Journal of Globl Optimiztion. 51 (2011) 743-751.

[27] J. Choi, C. C. Lim, A cholesky factorization based approach for blind FIR channel identification, IEEE Transaction on Signal Processing. 56 (2008) 1730-1735.

[28] H. Huang, F. Long, C. Li, Stabilization for a class of Markovian jump linear systems with linear fractional uncertainties, International Journal of Innovative Computing,Information and Control 11 (2015) 295-307.

[29] K. L. Teo, C. C. Lim, Time optimal control computation with application to ship steering, Journal of Optimization Theory and Applications 56 (1988) 145-156.

[30] X. X. Xu, C. C. Lim, Using transfer-resource graph for software-based verification of system-on-chip, IEEE Transactions on Computer-Aided Design of Integrated Circuits and Systems. 27 (2008) 1315-1328.

[31] L. Liu, Y. Shen, The asymptotic stability of nonlinear stochastic differential systems with Markovian switching and with polynomial growth, Journal of Mathematical Analysis and Applications, 391 (2012) 323-334.

[32] L. Liu, Y. Shen, New criteria on persistence in mean and extinction for stochastic competitive Lotka-Volterra systems with regime switching, Journal of Mathematical Analysis and Applications, 430 (2015) 306-323.

[33] Y. Liu, Z. Wang, J. Liang, and X. Liu, Synchronization of Coupled Neutral-Type Neural Networks With Jumping-Mode-Dependent Discrete and Unbounded Distributed Delays, IEEE Transactions on Systems, Man and Cybernetics-Part B: Cybernetic, 43 (2013) $102-114$.

[34] Y. Liu, Z. Wang, J. Liang and X. Liu, Stability and synchronization of discrete time Markovian jumping neural networks with mixed modedependent time-delays, IEEE Transactions on Neural Networks, 20 (2009) 1102-1116.

[35] H. Huang, D. W. C. Ho, and Y. Z. Qu, Robust stability of stochastic delayed additive neural networks with Markovian switching, Neural Network. 20, (2007) 799-809

[36] H. Huang, Y. Z. Qu, H. X. Li, Robust stability analysis of switched Hopfield neural networks with time-varying delay under uncertainty, Physics Letters A 345 (2005) 345-354.

[37] X. R. Mao, C. G. Yuan, Stochastic Differential Equations with Markovian Switching, Imperial College Press, London,U.K., 2006.

[38] A. Berman, R. J. Plemmons, Nonnegative matrices in the mathematical sciences, New York: Academic, 1979.

[39] A. Halanay, Differential Equations, Stability, Oscillation, Timelags, New York: Academic, 1996. 